\title{
FIRST INVENTORY OF VASCULAR FLORA OF MATOKIT MOUNTAIN (BIOKOVO MASSIF, CROATIA)
}

\section{PRVA INVENTARIZACIJA VASKULARNE FLORE PLANINE MATOKIT (BIOKOVO MASIV, HRVATSKA)}

Ivana VITASOVIĆ-KOSIĆ ${ }^{1 *}$, Mara VUKOJEVIĆ ${ }^{2}$ Sandro BOGDANOVIĆ ${ }^{1}$

\section{SUMMARY SAŽETAK}

The vascular flora of Matokit Mt (Biokovo Massif) in southern Croatia was researched in different vegetation periods from 2010-2015, and a total of 604 vascular plant taxa belonging to 86 families and 337 genera were found. The studied area has never been studied in the past and these are the first detailed floristic data about grasslands in different succession stages of Matokit Mt. Collected herbarium specimens ( 345 sheets) were digitalized and are available at the ZAGR Virtual Herbarium. The most dominant families were legumes (Fabaceae 9.9\%), grasses (Poaceae 9.1\%), daisies (Asteraceae 7.4\%) and mints (Lamiaceae 6.8\%). The analysis of life forms shows the dominance of hemicryptophytes (39.9\%) and therophytes (26.2\%) on Matokit Mt that indicates a high influence of the Mediterranean climate. A total of 36 endangered and 17 invasive plant taxa across the whole studied area were recorded. Endemic are 32 plant taxa (26 endemics in a broader sense and 6 stenoendemics) and they represent new site of Croatian flora. The occurrence of some very rare endemics (Cardamine fialae Fritsch and Erysimum croaticum Polatschek) in the flora of Matokit Mt is of special interest for the national flora.

KEY WORDS: flora diversity, endemic taxa, grassland succession, Vrgorac

\section{INTRODUCTION UVOD}

The Dinaric mountain range is the lower chained highland mountain of Southeastern Europe, representing the largest karst area that is known for its extremely high plant richness and biodiversity. The highest mountains in the Croatian Dinarides are Velebit, Dinara and Biokovo Massif. Within Biokovo Massif, Matokit Mt (lat. Monte Acutum, translated "the sharp Mountain") is the mountain ridge located in the surrounding of the town of Vrgorac in Dalmatian hinterland, and represents a wider area of the Biokovo Massif(Fig. 1). Matokit Mt provides the dinaric northwest- southeast direction with the highest peak (Sv. Rok) at 1062 $\mathrm{m}$ a.s.l. and total length of approximately $8.5 \mathrm{~km}$ (Vukosav 2006; Vukojević 2011).

Matokit Mt is situated in southern Croatia, next to the Vrgorac Town (Fig. 1). The eastern side of the Matokit Mt has relatively steep slopes, while the western slopes are easily accessible. The climate is a sub-Mediterranean with a great influence of continental climate, with an average annual temperature of $14.3^{\circ} \mathrm{C}$ (lower than the average of typical Mediterranean climate) and $1720 \mathrm{~mm}$ of average annual rainfall measured by National Hydrometeorological Institute from 1981 to 2010 for the Town Vrgorac). The duration of snow cover is short at a lower altitude, but in higher altitudinal zo-

\footnotetext{
${ }^{1}$ Doc. dr. sc. Ivana Vitasović-Kosić, University of Zagreb, Faculty of Agriculture, Department of Agricultural Botany, Svetošimunska 25, 10000 Zagreb, Croatia

${ }^{2}$ Mara Vukojević, mag. ing. agr., izv. prof. dr. sc. Sandro Bogdanović, Prapatnice 90, 21276 Vrgorac, Croatia

*Corresponding author: ivitasovic@agr.hr
} 

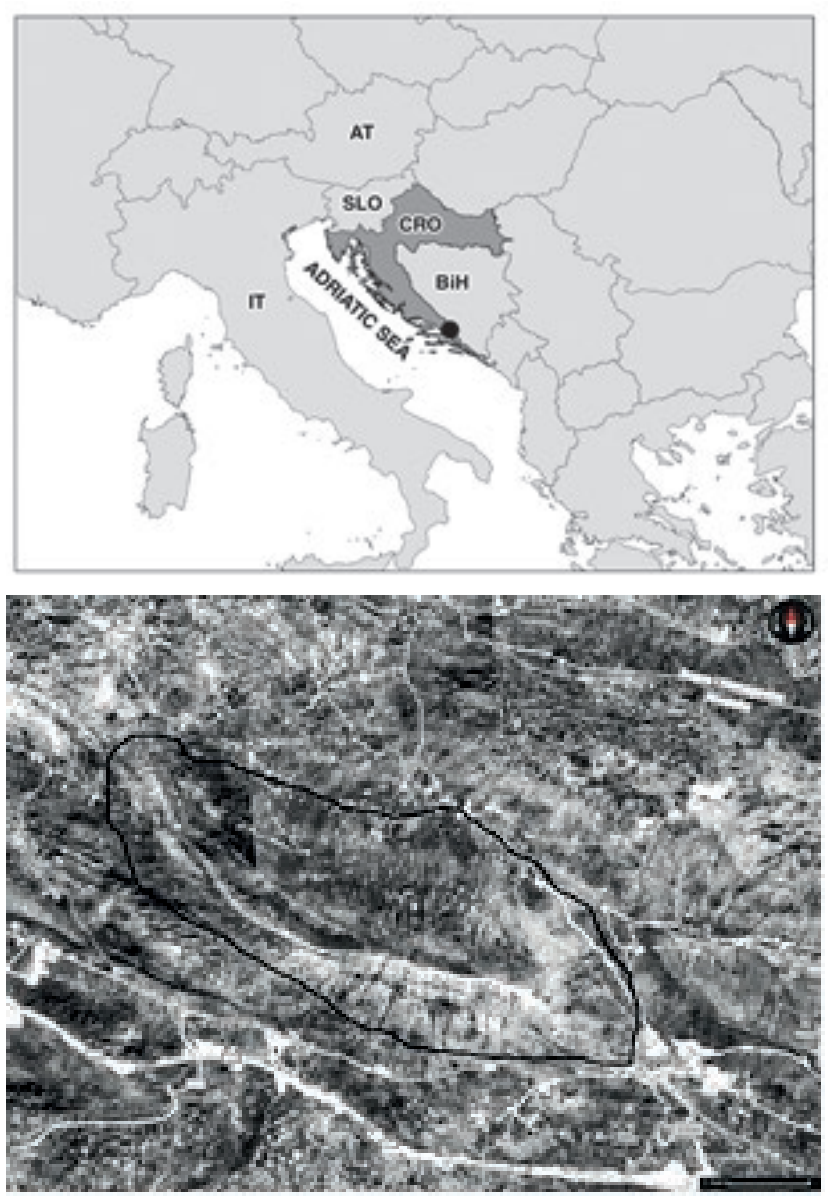

Figure 1. Geographical position of the researched area of Matokit Mt (Biokovo Massif)

Slika 1. Geografski smještaj istraživanog područja planine Matokit (Biokovo masiv)

nes the snow cover can last up to three months. In geological sense the northern slopes of Matokit Mt are composed of well-bedded (20-150 cm thick) Turonian limestone. Prevailing soils are shallow soils, with a significant share of stone fragments. In the areas between 500 and $1000 \mathrm{~m}$ a.s.l. there are brown soils (calcocambisol), and above $1000 \mathrm{~m}$ black soils on carbonate basis (calcomelanosol) (Martinović 2000).

In spite of the fact that botanical studies of broader area of Biokovo Massif started 60 years ago (Kušan 1956, 1969; Domac 1957; Radić 1974, 1976, 1977; Lovrić and Rac 1987; Rac and Lovrić 1987; Šilić and Šolić 1999, 2002; Pavletić 2002; Trinajstić 2002; Hršak and Alegro 2008; Alegro 2010), still botanical unexplored areas exist, and tharefore we founded necessary to fill this gap. The study area of Matokit Mt as a part of Biokovo Massif was chosen because its vascular flora has never been studied systematically in the past. Sporadically floristic records of invasive and threatened plant species of Matokit Mt were published by Vukojević (2011), Vukojević and Vitasović Kosić (2012) and Vukojević et al. (2016). Here we present the original results of the first comprehensive botanical study and flora inventory of Matokit $\mathrm{Mt}$, including its foothills, northern and southern slopes performed from 2010 to 2015.
The aim of this study was to fill the gap in botanical data of Matokit Mt as s part of Biokovo massif and to (a) perform inventory of the vascular flora, (b) to analyse plant diversity, as well as (c) to provide short overview on some interesting endemic and rare species of this mountain for species protection and conservation management of this area. The list of vascular flora includes an overview on some endemic, threatened, and invasive plant taxa of the Croatian flora.

\section{MATERIALS AND METHODS MATERIJALI I METODE}

\section{Study area - Područje istraživanja}

Field research of vascular flora was carried out on Matokit $\mathrm{Mt}$, as a part of Biokovo Massif, including its foothills (with nearby settlements), northern and southern slopes, the whole ridge of the mountain, covering different habitats,

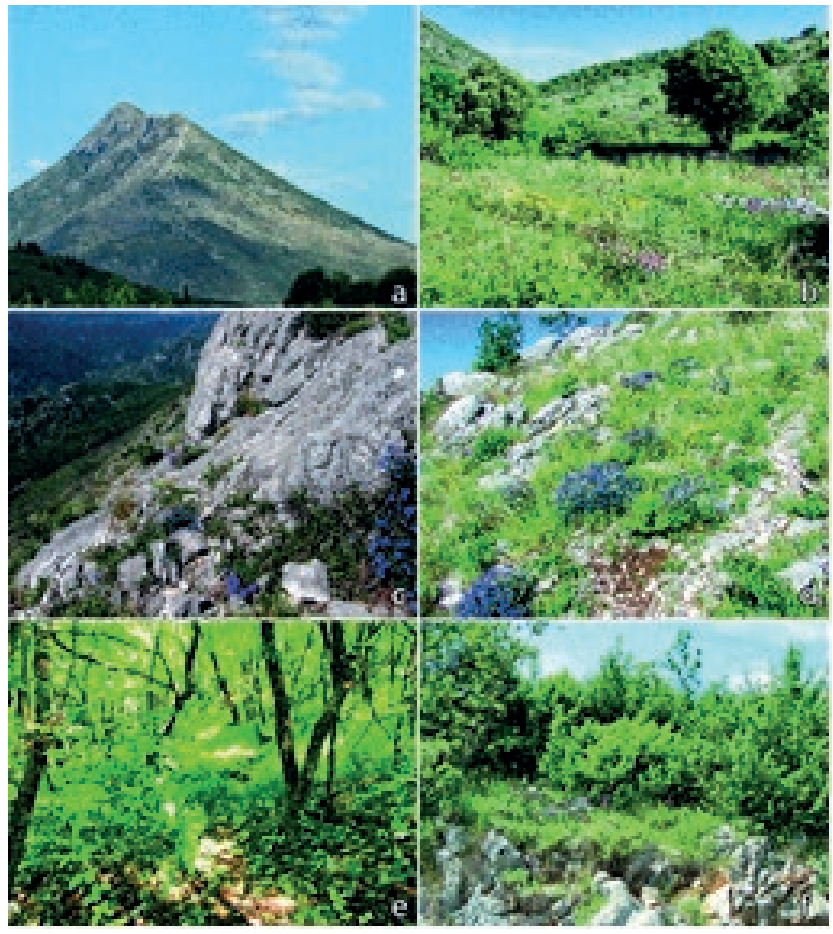

Figure 2. Matokit Mt (Biokovo Massif) and its habitats: a) peak ridge, b) submediterranean grasslands Saturejion subspicatae, c) rocky crevices with endemic Moltkia petraea (Tratt.) Griseb., and Edraianthus tenuifolius (Waldst. et Kit.) A. DC., d) rocky grasslands Saturejion subspicatae with domination of Ses/eria tenuifolia Schrad. ssp. tenuifolia and Moltkia petraea (Tratt.) Griseb., e) submediterranean forest of the alliance Ostryo-Carpinion orientalis, f) rocky grasslands and shrubland with stands of Juniperus oxycedrus L. (Photos by authors)

Slika 2. Planina Matokit (Biovski masiv) i njegova staništa: a) vršni greben, b) submediteranski travnjaci Saturejion subspicatae, c) stjenovite pukotine s endemičnim vrstama Moltkia petraea (Tratt.) Griseb. i Edraianthus tenuifolius (Waldst. Et Kit.) A. DC., d) kamenjarski travnjaci Saturejion subspicatae s dominacijom Sesleria tenuifolia Schrad. ssp. tenuifolia i Moltkia petraea (Tratt.) Griseb., e) submediteranska šuma sveze Ostryo-Carpinion orientalis, f) kamenjarski travnjaci i grmovite sastojine Juniperus oxycedrus L. (autorske fotografije) 
Table 1. Taxonomic analysis of the vascular flora of Matokit Mt (Biokovo Massif)

Tablica 1. Taksonomska analiza vaskularne flore planine Matokit (Biokovo masiv)

$\begin{array}{cccccc}\text { TAXA } & \text { PTERIDOPHYTA } & \text { Gymnospermae } & \text { Angiospermae } & \text { Lagnoliatae } & \text { Liliatae } \\ \text { Families } & 5 & 3 & 67 & 11 & 86 \\ \text { Genera } & 5 & 4 & 268 & 60 & 337 \\ \text { Species } & 8 & 5 & 456 & 102 & 569 \\ \text { Subspecies } & 0 & 2 & 23 & 10 & 35 \\ \text { Total no. of taxa } & 8 & 5 & 479 & 112 & 604 \\ \text { Total taxa (\%) } & 1.3 & 0.8 & 79.3 & 18.5 & 100\end{array}$

Table 2. Analysis of life forms and IUCN categories in the vascular flora of Matokit Mt (Biokovo Massif)

Tablica 2. Analiza životnih oblika i IUCN kategorija vaskularne flore planine Matokit (Biokovo masiv)

\begin{tabular}{|c|c|c|c|c|c|}
\hline $\begin{array}{l}\text { Life form } \\
\text { Životni oblik }\end{array}$ & $\begin{array}{l}\text { No. of taxa } \\
\text { Broj svojti }\end{array}$ & $\%$ & $\begin{array}{l}\text { IUCN category } \\
\text { IUCN kategorija }\end{array}$ & $\begin{array}{l}\text { No. of taxa } \\
\text { Broj svojti }\end{array}$ & $\begin{array}{c}\text { Endangered (\%) } \\
\text { Ugroženost }\end{array}$ \\
\hline $\mathrm{H}$ & 241 & 39.90 & NT & 16 & 44.44 \\
\hline$T$ & 158 & 26.16 & $\mathrm{DD}$ & 6 & 16.67 \\
\hline G & 75 & 12.42 & LC & 6 & 16.67 \\
\hline $\mathrm{Ph}$ & 65 & 10.76 & VU & 5 & 13.89 \\
\hline Ch & 65 & 10.76 & CR & 2 & 5.56 \\
\hline- & - & - & EN & 1 & 2.78 \\
\hline Total & 604 & 100.00 & Total & 36 & 100.00 \\
\hline
\end{tabular}

mostly grasslands in succession, rocky crevices and forest fringes, altogether covering about an area of $15 \mathrm{~km}^{2}$.

The researched area belongs to the Submediterranean zone (Fig. 2) with a high influence of the mountain climate and the vegetation is vertically divided according to altitude (from $250-1062 \mathrm{~m}$ a.s.l.). The dominant vegetation communities of Matokit Mt southern slopes, difficult to access, are dense maquis of alliance Fraxino orni-Quercion ilicis Biondi, Casavecchia et Gigante in Biondi et al. (2013), and large stands of Juniperus oxycedrus L., rising up to $600 \mathrm{~m}$ a.s.l. On the northern side the Matokit Mt is covered with forest vegetation that belongs to the thermophilous coastal forest and scrub alliance Fraxino orni-Ostryion Tomažič 1940 , with predominance of Querco-Carpinetum orientalis $\mathrm{H}$-ić 1939 in lower parts (up to $400 \mathrm{~m}$ a.s.l.) (Vukosav 2006; Trinajstić 2008; Vukojević et al. 2016).

\section{Data sources - Izvori podataka}

Data on plant taxa from Matokit Mt and it surroundings were collected during field researches from 2010 to 2015, throughout each vegetation season, using the standard methods for flora mapping in Croatia according to Nikolić (2006). Plant taxa were collected on two different transects (east-west and north-south) along the Matokit Mt, as well as in different types of habitats during four vegetation seasons on the same transects e.g. mountain routes. Herbar- ium specimens were collected, digitalized and deposited in the Herbarium ZAGR and are accessible through the Virtual Herbarium ZAGR on http://herbarium.agr.hr (Bogdanović et al. 2016).

Taxa were determined according to Pignatti $(1982,1984)$, Tutin et al. (1964-1980, 1993), and Nikolić (2019a). The plant nomenclature follows Flora Croatica Database (Nikolić 2019b). The plant taxa listed in Tab. 3 are given in alphabetical order where the families and life forms were attributed to each taxon, IUCN category, endemic status, or invasiveness are also provided. The attributed life forms were denoted according to classification of Raunkiaer (1934) and Horvat (1949). In the floristic list (Tab. 3) they were marked with the following abbreviations Ch (Chamaephyta), G (Geophyta), $\mathrm{H}$ (Hemicryptophyta), Ph (Phanerophyta), and $\mathrm{T}$ (Therophyta). The status of endangered plant taxa was analysed according to IUCN criteria and categories that are implemented in the Flora Croatica Database (Nikolić 2019b), thereby the following abbreviations were used: $\mathrm{CR}$ (Critically endangered), EN (Endangered), VU (Vulnerable), LC (Least Concern), NT (Near Threatened), and DD (Data Deficient). Endemic status of plant taxa, endemic (E) and stenoendemic (sE) was denoted according to Nikolić et al. (2015) and the Flora Croatica Database (Nikolić 2019b). The status of invasive alien species (Inv) was denoted according to Boršić et al. (2008) and Nikolić et al. (2014). 


\section{RESULTS AND DISCUSSION REZULTATI I RASPRAVA}

During the floristic research of Matokit Mt, a total of 604 vascular plant taxa were found (Tab. 3). In taxonomic analysis 569 species and 35 subspecies of native and naturalised vascular plants were noted, altogether from 86 families and 337 genera. Eight taxa of Pteridophyta, five taxa of Gymnospermae, and 591 taxa of Angiospermae (Magnoliatae 479 and Liliatae 112 taxa) were recorded (Tab. 1). The most represented families were Fabaceae (9.9\%), Poaceae (9.1\%), Asteraceae (7.4\%), Lamiaceae (6.8\%), Brassicaceae (5.0\%) and Rosaceae (4.8\%). A total of 345 herbarium sheets were collected and deposited at the ZAGR herbarium (206 of them have been digitalized till now).

The analysis of life forms (Tab. 2) indicate that dominant life forms on Matokit Mt are hemicryptophytes (39.9\%) and therophytes (26.2\%). Geophytes are represented with $12.4 \%$, while phanerophytes and chamaephytes with $10.8 \%$ respectively.

Table 3. Alphabetical list of vascular flora of Matokit Mt (Biokovo Massif). Tablica 3. Abecedni popis vaskularne flore planine Matokit (Biokovo masiv)

1. Acanthus balcanicus Heywood et I. Richardson, Acanthaceae, $\mathrm{H}$

2. Acer monspessulanum L., Aceraceae, Ph

3. Achillea millefolium L., Asteraceae, $\mathrm{H}$

4. Acinos arvensis (Lam.) Dandy, Lamiaceae, Ch

5. Aegilops geniculata Roth, Poaceae, T

6. Aegilops neglecta Req. ex Bertol., Poaceae, T, NT

7. Aethionema saxatile (L.) R. Br., Brassicaceae, Ch

8. Agrimonia eupatoria L., Rosaceae, $\mathrm{H}$

9. Agrostemma githago L., Caryophyllaceae, T

10. Ailanthus altissima (Mill.) Swingle, Simaroubaceae, $\mathrm{Ph}$, Inv

11. Aira elegantissima Schur, Poaceae, $\mathrm{T}$

12. Ajuga chamaepitys (L.) Schreb., Lamiaceae, T

13. Ajuga genevensis L., Lamiaceae, $\mathrm{H}$

14. Ajuga reptans $\mathrm{L}$, Lamiaceae, $\mathrm{H}$

15. Alliaria petiolata (M. Bieb.) Cavara et Grande, Brassicaceae, $\mathrm{H}$

16. Allium ampeloprasum $\mathrm{L}$., Amaryllidaceae, $\mathrm{G}$

17. Allium carinatum $\mathrm{L}$., Amaryllidaceae, $\mathrm{G}$

18. Allium deutiferum Webb et Berthel., Amaryllidaceae, $G$

19. Allium flavum L., Amaryllidaceae, $\mathrm{G}$

20. Allium lusitanicum Lam., Amaryllidaceae, $\mathrm{G}$

21. Allium moschatum L., Amaryllidaceae, $G$

22. Allium pallens L. ssp. tenuiflorum (Ten.) Stearn, Amaryllidaceae, $G$

23. Allium roseum L., Amaryllidaceae, $\mathrm{G}$

24. Allium spherocephalon L., Amaryllidaceae, G

25. Alyssoides utriculata (L.) Medik., Brassicaceae, Ch

26. Alyssum murale Waldst. et Kit., Brassicaceae, Ch

27. Amaranthus retroflexus L., Amaranthaceae, T, Inv

28. Ambrosia artemisiifolia L., Asteraceae, T, Inv,

29. Amelanchier ovalis Medik., Rosaceae, N

30. Anacamptis pyramidalis (L.) Rich., Orchidaceae, G, NT

31. Anagallis arvensis L., Primulaceae, T

32. Anagallis coerulea Schreb., Primulaceae, $\mathrm{T}$

33. Anchusella cretica (Mill.) Bigazzi, E.Nardi et Salvi, Boraginaceae, T

34. Anthericum liliago L., Asparagaceae, G

35. Anthoxanthum odoratum L., Poaceae, $\mathrm{H}$

36. Anthriscus sylvestris (L.) Hoffm., Apiaceae, $\mathrm{H}$
37. Anthyllis vulneraria L., Fabaceae, $\mathrm{H}$

38. Anthyllis vulneraria L. subsp. pulchella (Vis.) Bornm., Fabaceae, $\mathrm{H}$

39. Aposeris foetida (L.) Less., Asteraceae, $\mathrm{H}$

40. Arabis collina Ten., Brassicaceae, $\mathrm{H}$

41. Arabis glabra (L.) Bernhardt, Brassicaceae, $\mathrm{H}$

42. Arabis hirsuta (L.) Scop., Brassicaceae, $\mathrm{H}$

43. Arabis turrita L., Brassicaceae, $\mathrm{H}$

44. Arabis verna (L.) R. Br., Brassicaceae, T

45. Argyrolobium zanonii (Turra) P. W. Ball, Fabaceae, Ch

46. Aristolochia rotunda L., Aristolochiaceae, G

47. Artemisia vulgaris L., Asteraceae, Ch

48. Arum italicum Mill., Araceae, G

49. Asparagus acutifolius L., Asparagaceae, N

50. Asperula aristata L.f., Rubiaceae, $\mathrm{H}$

51. Asperula scutellaris Vis., Rubiaceae, Ch, E

52. Asphodeline lutea (L.) Reichenb., Xanthorrhoeaceae, G

53. Asplenium ceterach L., Aspleniaceae, $\mathrm{H}$

54. Asplenium onopteris L., Aspleniaceae, $\mathrm{H}$

55. Asplenium ruta-muraria L., Aspleniaceae, $\mathrm{H}$

56. Asplenium trichomanes L., Aspleniaceae, $\mathrm{H}$

57. Astragalus muelleri Steud. et Hochst., Fabaceae, H, NT, E

58. Astragalus vesicarius L., Fabaceae, Ch

59. Asyneuma limonifolium (L.) Janch., Campanulaceae, H

60. Athyrium filix-femina (L.) Roth, Woodsiaceae, $\mathrm{H}$

61. Avena barbata Pott ex Link, Poaceae, T

62. Bellis annua L., Asteraceae, T

63. Bellis perennis L., Asteraceae, $\mathrm{H}$

64. Bellis sylvestris Cirillo, Asteraceae, $\mathrm{H}$

65. Berteroa mutabilis (Vent.) DC., Brassicaceae, $\mathrm{H}$

66. Betonica officinalis L., Lamiaceae, $\mathrm{H}$

67. Bituminaria bituminosa (L.) Stirton, Fabaceae, H

68. Blackstonia perfoliata (L.) Huds., Gentianaceae, T

69. Bombycilaena erecta (L.) Smoljan., Asteraceae, T

70. Brachypodium distachyon (L.) P. Beauv., Poaceae, T

71. Brachypodium pinnatum (L.) P. Beauv., Poaceae, $\mathrm{H}$

72. Brachypodium sylvaticum (Huds.) P. Beauv., Poaceae, H

73. Brassica oleracea L. ssp. acephala (DC.) O. Schwarz, Brassicaceae, Ch

74. Briza maxima L., Poaceae, T

75. Briza minor L., Poaceae, T

76. Bromus commutatus Schrad., Poaceae, T

77. Bromus erectus Huds., Poaceae, $\mathrm{H}$

78. Bromus hordeaceus L., Poaceae, $\mathrm{T}$

79. Bromus squarrosus L., Poaceae, T

80. Bromus sterilis L., Poaceae, T

81. Broussonetia papyrifera (L.) Vent., Moraceae, Ph, Inv

82. Bryonia dioica Jacq., Cucurbitaceae, $\mathrm{H}$

83. Bunium alpinum Waldst. et Kit. ssp. montanum (W. D. J. Koch) P. W. Ball, Apiaceae, Ch

84. Bupleurum praealtum L., Apiaceae, T

85. Bupleurum veronense Turra, Apiaceae, $T$

86. Calamagrostis epigejos (L.) Roth, Poaceae, $\mathrm{H}$

87. Calamintha grandiflora (L.) Moench, Lamiaceae, H

88. Calamintha nepetoides Jord., Lamiaceae, H

89. Calamintha sylvatica Bromf., Lamiaceae, $\mathrm{H}$

90. Campanula bononiensis L., Campanulaceae, $\mathrm{H}$

91. Campanula lingulata Waldst. et Kit., Campanulaceae, H

92. Campanula persicifolia L., Campanulaceae, $\mathrm{H}$

93. Campanula pyramidalis L., Campanulaceae, $\mathrm{H}$

94. Campanula rapunculoides L., Campanulaceae, $\mathrm{H}$

95. Campanula rapunculus L., Campanulaceae, $\mathrm{H}$

96. Campanula sibirica L., Campanulaceae, $\mathrm{H}$

97. Capsella bursa-pastoris (L.) Medik., Brassicaceae, H

98. Capsella rubella Reut., Brassicaceae, T

99. Capsicum annuum L., Solanaceae, T

100. Cardamine fialae Fritsch, Brassicaceae, T, sE 
101. Cardamine graeca L., Brassicaceae, $T$

102. Cardamine hirsuta L., Brassicaceae, $H$

103. Cardaria draba (L.) Desv., Brassicaceae, H

104. Carduus micropterus (Borbás) Teyber, Asteraceae, H

105. Carduus pycnocephalus L., Asteraceae, T

106. Carex caryophyllea Latourr., Cyperaceae, $\mathrm{H}$

107. Carex divulsa Stokes, Cyperaceae, H

108. Carex flacca Schreb., Cyperaceae, G

109. Carex hallerana Asso, Cyperaceae, $\mathrm{H}$

110. Carex humilis Leyss., Cyperaceae, $\mathrm{H}$

111. Carex muricata L. agg., Cyperaceae, $\mathrm{H}$

112. Carex spicata Huds., Cyperaceae, $\mathrm{H}$

113. Carlina corymbosa L., Asteraceae, $\mathrm{H}$

114. Carpinus orientalis Mill., Corylaceae, $\mathrm{Ph}$

115. Celtis australis L., Ulmaceae, $\mathrm{Ph}$

116. Centaurea cyanus L., Asteraceae, T

117. Centaurea deusta Ten., Asteraceae, $\mathrm{H}$

118. Centaurea deusta Ten. ssp. concolor (DC.) Hayek, Asteraceae, H

119. Centaurea glaberrima Tausch, Asteraceae, H, NT, sE

120. Centaurea pannonica (Heuff.) Simonk., Asteraceae, H

121. Centaurea rupestris L., Asteraceae, $\mathrm{H}$

122. Centaurium erythraea Rafn, Gentianaceae, $\mathrm{T}$

123. Cephalaria leucantha (L.) Roem. et Schult., Dipsacaceae, H

124. Cerastium dubium (Bast.) Guépin, Caryophyllaceae, T

125. Cerastium grandiflorum Waldst. et Kit., Caryophyllaceae, Ch, E

126. Cerastium pumilum Curtis, Caryophyllaceae, $T$

127. Cerastium pumilum Curtis ssp. glutinosum (Fries) Jalas, Caryophyllaceae, T

128. Cercis siliquastrum L., Fabaceae, Ph

129. Chaenorhinum minus (L.) Lange, Scrophulariaceae, $T$

130. Chaenorhinum minus (L.) Lange ssp. litorale (Willd.) Hayek, Scrophulariaceae, T

131. Chaerophyllum coloratum L., Scrophulariaceae, T, NT, E

132. Chaerophyllum temulum L., Scrophulariaceae, $T$

133. Cheilanthes acrostica (Balbis) Tod., Adianthaceae, $\mathrm{H}$

134. Chelidonium majus L., Papaveraceae, $\mathrm{H}$

135. Chenopodium album L., Chenopodiaceae, $T$

136. Chenopodium vulvaria L., Chenopodiaceae, T, DD

137. Chondrilla juncea L., Cichoriaceae, $\mathrm{H}$

138. Chrysopogon gryllus (L.) Trin., Poaceae, H

139. Cichorium intybus L., Cichoriaceae, $\mathrm{H}$

140. Cirsium acaule Scop., Asteraceae, H

141. Cirsium arvense (L.) Scop., Asteraceae, G

142. Cirsium vulgare (Savi) Ten., Asteraceae, H

143. Cistus incanus L., Fabaceae, N

144. Cistus salvifolius L., Fabaceae, N

145. Cleistogenes serotina (L.) Keng, Poaceae, H

146. Clematis flammula L., Ranunculaceae, $\mathrm{Ph}$

147. Clematis recta L., Ranunculaceae, H

148. Clematis vitalba L., Ranunculaceae, Ph

149. Clematis viticella L., Ranunculaceae, $\mathrm{H}$

150. Clinopodium vulgare $L .$, Lamiaceae, $H$

151. Colchicum autumnale L., Colchicaceae, G

152. Colchicum hungaricum Janka, Colchicaceae, G

153. Colutea arborescens L., Fabaceae, N

154. Consolida ajacis (L.) Schur, Ranunculaceae, T, CR

155. Convolvulus arvensis L., Convolvulaceae, G

156. Convolvulus cantabrica L., Convolvulaceae, $\mathrm{H}$

157. Convolvulus tricolor L., Convolvulaceae, $T$

158. Conyza canadensis (L.) Cronquist, Asteraceae, T, Inv

159. Cornus mas L., Cornaceae, Ph

160. Cornus sanguinea L., Cornaceae, $\mathrm{Ph}$

161. Coronilla emerus L. ssp. emeroides Boiss. et Spruner, Fabaceae, N

162. Coronilla scorpioides (L.) Koch, Fabaceae, T

163. Coronilla varia L., Fabaceae, $\mathrm{H}$

164. Corydalis solida (L.) Swartz, Papaveraceae, G

165. Cotinus coggygria Scop., Anacardiaceae, N
166. Crataegus monogyna Jacq., Rosaceae, $\mathrm{Ph}$

167. Crepis biennis L., Cichoriaceae, $\mathrm{H}$

168. Crepis capillaris (L.) Wallr., Cichoriaceae, $T$

169. Crepis sancta (L.) Babc., Cichoriaceae, $T$

170. Crepis vesicaria L., Cichoriaceae, T

171. Crocus reticulatus Steven ex Adams, Iridaceae, $G$

172. Crocus vernus (L.) Hill, Iridaceae, G

173. Crocus vernus (L.) Hill ssp. albiflorus (Kit.) Asch. et Graebn., Iridaceae, G

174. Cruciata glabra (L.) Ehrend., Rubiaceae, $\mathrm{H}$

175. Cruciata laevipes Opiz, Rubiaceae, $\mathrm{H}$

176. Cupressus sempervirens L., Cupressaceae, $\mathrm{Ph}$

177. Cuscuta campestris Yuncker, Cuscutaceae, T, Inv

178. Cyclamen hederifolium Aiton, Primulaceae, G

179. Cyclamen repandum Sibth. et Sm., Primulaceae, G, NT

180. Cymbalaria muralis P. Gaertn., B. Mey. et Scherb., Scrophulariaceae, T

181. Cynara scolymus L., Asteraceae, $\mathrm{H}$

182. Cynodon dactylon (L.) Pers., Poaceae, G

183. Cynoglossum columnae Ten., Boraginaceae, $T$

184. Cynoglossum officinale L., Boraginaceae, $\mathrm{H}$

185. Cynosurus echinatus L., Poaceae, T

186. Dactylis glomerata L., Poaceae, $\mathrm{H}$

187. Dactylis glomerata L. ssp. hispanica (Roth) Nyman, Poaceae, H

188. Dasypyrum villosum (L.) P.Candargy, Poaceae, T

189. Daucus carota L., Apiaceae, H

190. Desmazeria rigida (L.) Tutin, Poaceae, T

191. Dianthus ciliatus Guss. ssp. dalmaticus (Čelak.) Hayek, Caryophyllaceae, H, E

192. Dianthus sylvestris Wulfen in Jacq., Caryophyllaceae, $\mathrm{H}$

193. Dianthus sylvestris Wulfen in Jacq. ssp. tergestinus (Rchb.) Hayek, Caryophyllaceae, $\mathrm{H}, \mathrm{E}$

194. Dichanthium ischaemum (L.) Roberty, Poaceae, $\mathrm{H}$

195. Dictamnus albus L., Rutaceae, H

196. Digitaria sanguinalis (L.) Scop., Poaceae, T

197. Diplotaxis viminea (L.) DC., Brassicaceae, $T$

198. Dorycnium germanicum (Gremli) Rikli, Fabaceae, Ch

199. Draba muralis L., Brassicaceae, T

200. Ecballium elaterium (L.) A. Rich., Cucurbitaceae, G, DD

201. Echium italicum L., Boraginaceae, T

202. Edraianthus tenuifolius (Waldst. et Kit.) A. DC., Campanulaceae, Ch, E

203. Elymus repens (L.) Gould, Poaceae, G

204. Ephedra fragilis Desf. ssp. campylopoda (C. A. Mayer) Asch. et Graeb.,

Epherdaceae, N, NT

205. Epilobium angustifolium L., Onagraceae, $\mathrm{H}$

206. Epilobium ciliatum Raf., Onagraceae, $\mathrm{H}$, Inv

207. Epilobium dodonaei Vill., Onagraceae, $\mathrm{H}$

208. Erigeron annuus (L.) Pers., Asteraceae, $\mathrm{H}$, Inv

209. Eriobotrya japonica (Thunb.) Lindl., Rosaceae, N

210. Erodium cicutarium (L.) L' Hér., Geraniaceae, T

211. Eryngium amethystinum L., Apiaceae, $\mathrm{H}$

212. Eryngium campestre L., Apiaceae, $\mathrm{H}$

213. Erysimum croaticum Polatschek, Brassicaceae, $\mathrm{H}, \mathrm{sE}$

214. Erythronium dens-canis L., Liliaceae, G

215. Euonymus verrucosa Scop., Celastraceae, N

216. Euphorbia characias L. ssp. wulfenii (Hoppe ex Koch) A. M. Sm., Euphorbiaceae, N

217. Euphorbia cyparissias L., Euphorbiaceae, $\mathrm{H}$

218. Euphorbia falcata L., Euphorbiaceae, T

219. Euphorbia fragifera Jan, Euphorbiaceae, Ch

220. Euphorbia myrsinites L., Euphorbiaceae, Ch

221. Euphorbia prostrata Aiton, Euphorbiaceae, T

222. Euphorbia spinosa L., Euphorbiaceae, Ch

223. Fallopia convolvulus (L.) Á.Löve, Polygonaceae, T

224. Ficus carica L., Moraceae, $\mathrm{Ph}$

225. Filago lutescens Jord. agg., Asteraceae, $T$

226. Filipendula vulgaris Moench, Rosaceae, $\mathrm{H}$

227. Foeniculum vulgare Mill., Apiaceae, G

228. Fragaria vesca L., Rosaceae, $\mathrm{H}$ 
229. Frangula alnus Mill., Rhamnaceae, $\mathrm{Ph}$

230. Frangula rupestris (Scop.) Schur., Rhamnaceae, $\mathrm{N}$

231. Fraxinus ornus L., Oleaceae, $\mathrm{Ph}$

232. Fritillaria orientalis Adams, Liliaceae, G

233. Fumana ericifolia Wallr., Cistaceae, Ch

234. Fumana procumbens (Dunal) Gren. et Godr., Cistaceae, Ch

235. Fumaria officinalis $L$., Fumariaceae, $T$

236. Gagea spathacea (Hayne) Salisb., Liliaceae, G

237. Galeopsis angustifolia Hoffm., Lamiaceae, T

238. Galinsoga parviflora Cav., Asteraceae, T

239. Galium album Mill., Rubiaceae, H

240. Galium aparine L., Rubiaceae, T

241. Galium corrudifolium Vill., Rubiaceae, H

242. Galium firmum Tausch, Rubiaceae, H, E

243. Galium mollugo L., Rubiaceae, $\mathrm{H}$

244. Galium verum L., Rubiaceae, $H$

245. Genista januensis Viv., Fabaceae, Ch

246. Genista pilosa L., Fabaceae, Ch

247. Genista sericea Wulfen, Fabaceae, Ch, E

248. Genista sylvestris Scop., Fabaceae, Ch

249. Genista sylvestris Scop. ssp. dalmatica (Bartl.) H. Lindb., Fabaceae, Ch, E

250. Geranium columbinum L., Geraniaceae, T

251. Geranium dissectum L., Geraniaceae, $T$

252. Geranium lucidum L., Geraniaceae, $T$

253. Geranium macrorrhizum L., Geraniaceae, $H$

254. Geranium molle L., Geraniaceae, T

255. Geranium robertianum L., Geraniaceae, $T$

256. Geranium sanguineum L., Geraniaceae, $\mathrm{H}$

257. Geum urbanum L., Rosaceae, $\mathrm{H}$

258. Gladiolus communis L., Iridaceae, $G$

259. Gladiolus illyricus W. D. J. Koch, Iridaceae, G

260. Gladiolus italicus Mill., Iridaceae, G

261. Glechoma hirsuta Waldst. et Kit., Lamiaceae, H

262. Globularia cordifolia L., Globulariaceae, Ch

263. Globularia cordifolia L. ssp. bellidifolia (Ten.) Wettst., Globulariaceae, Ch

264. Haplophyllum patavinum (L.) G. Don, Rutaceae, Ch

265. Hedera helix L., Araliaceae, Ph

266. Helianthemum canum (L.) Baumg., Cistaceae, Ch

267. Helianthemum nummularium (L.) Mill. , Cistaceae, Ch

268. Helianthemum nummularium (L.) Mill. ssp. grandiflorum (Scop.) Schinz et Thell., Cistaceae, Ch

269. Helianthemum oelandicum (L.) DC. ssp. alpestre (Jacq.) Breistr., Cistaceae, Ch 270. Helianthus tuberosus L., Asteraceae, G, Inv

271. Helichrysum italicum (Roth) G. Don, Asteraceae, Ch

272. Heliotropium europaeum L., Boraginaceae, $T$

273. Hermodactylus tuberosus (L.) Mill., Iridaceae, G

274. Herniaria glabra L., Caryophyllaceae, T

275. Hesperis laciniata All., Brassicaceae, $\mathrm{H}$

276. Hibiscus trionum L., Malvaceae, T, EN

277. Hieracium pilosella L., Cichoriaceae, $\mathrm{H}$

278. Hieracium praealtum Vill. ex Gochnat ssp. bauhinii (Besser) Petunn., Cichoriaceae, $\mathrm{H}$

279. Hordeum murinum L. , Poaceae, T

280. Hordeum murinum L. ssp. leporinum (Link) Arcang., Poaceae, $T$

281. Hypericum perforatum L., Clusiaceae, $\mathrm{H}$

282. Inula britannica L. agg., Asteraceae, $\mathrm{H}$

283. Inula conyza DC., Asteraceae, $\mathrm{H}$

284. Inula ensifolia L., Asteraceae, $\mathrm{H}$

285. Inula oculus-christi L., Asteraceae, $\mathrm{H}$

286. Inula salicina L. , Asteraceae, $\mathrm{H}$

287. Inula spiraeifolia L., Asteraceae, $\mathrm{H}$

288. Inula verbascifolia (Willd.) Hausskn., Asteraceae, Ch

289. Iris illyrica Tomm., Iridaceae, G, LC, E

290. Iris pseudopallida Trinajstić , Iridaceae, G, E

291. Juncus articulatus L., Juncaceae, $\mathrm{H}$
292. Juniperus oxycedrus L., Cupressaceae, $N$

293. Jurinea mollis (L.) Rchb., Asteraceae, H

294. Kickxia commutata (Bernh. ex Rchb.) Fritsch agg., Scrophulariaceae, Ch

295. Knautia arvensis (L.) Coult., Dipsacaceae, $\mathrm{H}$

296. Koeleria pyramidata (Lam.) P.Beauv., Poaceae, $\mathrm{H}$

297. Koeleria splendens C. Presl, Poaceae, H

298. Lactuca perennis L., Cichoriaceae, $\mathrm{H}$

299. Lactuca saligna L., Cichoriaceae, $T$

300. Lactuca serriola L., Cichoriaceae, $\mathrm{H}$

301. Lactuca viminea (L.) J. et C. Presl, Cichoriaceae, H

302. Lactuca virosa L., Cichoriaceae, $T$

303. Lamium amplexicaule L., Lamiaceae, T

304. Lamium maculatum L., Lamiaceae, $\mathrm{H}$

305. Lamium orvala L., Lamiaceae, $\mathrm{H}$

306. Lapsana communis L., Cichoriaceae, $\mathrm{T}$

307. Laserpitium siler L., Apiaceae, H

308. Lathyrus aphaca L., Fabaceae, T

309. Lathyrus cicera L., Fabaceae, $T$

310. Lathyrus latifolius L., Fabaceae, H

311. Lathyrus sphaericus Retz., Fabaceae, $T$

312. Lathyrus vernus (L.) Bernhardt, Fabaceae, $\mathrm{H}$

313. Legousia speculum-veneris (L.) Chaix, Campanulaceae, T

314. Ligustrum vulgare L., Oleaceae, $\mathrm{N}$

315. Lilium martagon L., Liliaceae, G, VU

316. Lilium martagon L. ssp. cattaniae (Vis.) Degen, Liliaceae, G, sE

317. Limodorum abortivum (L.) Sw., Orchidaceae, G

318. Linaria microsepala A. Kern., Scrophulariaceae, G, DD, sE

319. Linaria vulgaris Mill., Scrophulariaceae, G, E

320. Linum nodiflorum L., Linaceae, $T$

321. Linum perenne L., Linaceae, $H$

322. Linum tenuifolium L., Linaceae, $\mathrm{Ch}$

323. Lithospermum purpurocaeruleum L., Boraginaceae, Ch

324. Lobularia maritima (L.) Desv., Brassicaceae, $\mathrm{H}$

325. Lolium multiflorum Lam., Poaceae, T

326. Lolium perenne L., Poaceae, H

327. Lonicera etrusca Santi, Caprifoliaceae, N

328. Lotus corniculatus L., Fabaceae, $\mathrm{H}$

329. Lunaria annua L., Brassicaceae, $T$

330. Lunaria rediviva L., Brassicaceae, $\mathrm{H}$

331. Luzula campestris (L.) DC. agg., Poaceae, $\mathrm{H}$

332. Luzula forsteri (Sm.) DC., Poaceae, H

333. Malus domestica Borkh., Rosaceae, Ph

334. Malva alcea L., Malvaceae, H

335. Malva moschata L., Malvaceae, H

336. Malva nicaeensis All., Malvaceae, T

337. Malva sylvestris L., Malvaceae, $\mathrm{H}$

338. Marrubium incanum Desr., Lamiaceae, H

339. Marrubium vulgare L., Lamiaceae, Ch

340. Matricaria perforata Mérat, Asteraceae, T

341. Matthiola incana (L.) R. Br., Brassicaceae, Ch, NT

342. Medicago arabica (L.) Huds., Fabaceae, T

343. Medicago falcata L., Fabaceae, H

344. Medicago lupulina L., Fabaceae, T

345. Medicago minima (L.) Bartal, Fabaceae, T

346. Medicago orbicularis (L.) Bartal., Fabaceae, T

347. Medicago prostrata Jacq., Fabaceae, H

348. Medicago sativa L., Fabaceae, H

349. Melica ciliata L., Poaceae, H

350. Melilotus italica (L.) Lam., Fabaceae, T

351. Melissa officinalis L., Lamiaceae, $\mathrm{H}$

352. Melittis melissophyllum L., Lamiaceae, $\mathrm{H}$

353. Mentha longifolia (L.) Huds., Lamiaceae, H

354. Mercurialis annua L., Euphorbiaceae, T

355. Meum athamanticum Jacq., Apiaceae, $\mathrm{H}$

356. Micromeria juliana (L.) Benth. ex Rchb., Lamiaceae, Ch 
357. Moenchia mantica (L.) Bartl., Caryophyllaceae, T

358. Moltkia petraea (Tratt.) Griseb., Boraginaceae, H, E

359. Morus alba L., Moraceae, Ph

360. Morus nigra L., Moraceae, Ph

361. Muscari botryoides (L.) Mill., Asparagaceae, G

362. Muscari comosum (L.) Mill., Asparagaceae, G

363. Muscari neglectum Guss. ex Ten., Asparagaceae, G

364. Mycelis muralis (L.) Dumort., Cichoriaceae, $\mathrm{H}$

365. Myosotis arvensis (L.) Hill, Boraginaceae, T

366. Myosotis ramosissima Rochel, Boraginaceae, $\mathrm{T}$

367. Myrrhoides nodosa (L.) Cannon, Apiaceae, T

368. Narcissus radiiflorus Salisb., Amaryllidaceae, G

369. Neottia nidus-avis (L.) Rich., Orchidaceae, G

370. Nigella damascena L., Ranunculaceae, T

371. Odontites lutea (L.) Clairv., Scrophulariaceae, T

372. Oenothera biennis L., Onagraceae, H, Inv

373. Ononis pusilla L., Fabaceae, H

374. Ononis spinosa L., Fabaceae, Ch

375. Onopordum illyricum L., Asteraceae, $\mathrm{H}$

376. Onosma echioides (L.) L. ssp. dalmatica (Scheele) Peruzziet N. G. Passal.,

Boraginaceae, $\mathrm{Ch}, \mathrm{E}$

377. Ophrys sphegodes Mill., Orchidaceae, G, VU

378. Opopanax chironium (L.) Koch, Apiaceae, $\mathrm{H}$

379. Orchis coriophora L., Orchidaceae, G, VU

380. Orchis morio L., Orchidaceae, G, NT

381. Orchis provinciallis Balb. ssp. pauciflora (Ten.) Camus, Orchidaceae, G

382. Orchis quadripunctata Cirillo ex Ten., Orchidaceae, G, VU

383. Orchis tridentata Scop., Orchidaceae, G, VU

384. Origanum majorana L., Lamiaceae, $\mathrm{H}$

385. Origanum vulgare L. , Lamiaceae, Ch

386. Orlaya grandiflora (L.) Hoffm., Apiaceae, T

387. Ornithogalum comosum L., Asparagaceae, G

388. Ornithogalum pyramidale L., Asparagaceae, G

389. Ornithogalum umbellatum L., Asparagaceae, G

390. Orobanche sp., Orobanchaceae, G

391. Ostrya carpinifolia Scop., Corylaceae, Ph

392. Osyris alba L., Santalaceae, N

393. Paliurus spina-christi Mill., Rhamnaceae, N

394. Papaver argemone L., Papaveraceae, T, CR

395. Papaver rhoeas L., Papaveraceae, $\mathrm{T}$

396. Parietaria judaica L., Urticaceae, $\mathrm{H}$

397. Paronychia kapela (Hacq.) A. Kerner, Caryophyllaceae, H

398. Parthenocissus quinquefolia (L.) Planchon, Vitaceae, Ph, Inv

399. Peltaria alliacea Jacq., Brassicaceae, H, NT, E

400. Petrorhagia prolifera (L.) P.W. Ball et Heywood, Caryophyllaceae, T

401. Petrorhagia saxifraga (L.) Link, Caryophyllaceae, H

402. Peucedanum oreoselinum (L.) Moench, Apiaceae, H

403. Peucedanum ostruthium (L.) Koch, Apiaceae, $\mathrm{H}$

404. Phalaris paradoxa L., Poaceae, T, DD

405. Phillyrea latifolia L., Oleaceae, Ph

406. Phleum echinatum Host, Poaceae, T

407. Phleum subulatum (Savi) Asch. et Graebn., Poaceae, $T$

408. Physalis alkekengi L., Solanaceae, H

409. Phytolacca americana L., Phytolaccaceae, G, Inv

410. Picris echioides L., Cichoriaceae, T

411. Picris hieracioides L., Cichoriaceae, $\mathrm{H}$

412. Pimpinella major (L.) Huds., Apiaceae, H

413. Pinus halepensis Mill., Pinaceae, Ph

414. Pinus nigra Arnold ssp. dalmatica (Vis.) Franco, Pinaceae, Ph, NT, sE

415. Pistacia terebinthus L., Anacardiaceae, Ph

416. Plantago lanceolata L., Plantaginaceae, H

417. Plantago media L., Plantaginaceae, $\mathrm{H}$

418. Platanthera chlorantha (Custer) Rchb., Orchidaceae, G, NT

419. Plumbago europaea L., Plumbaginaceae, Ch

420. Poa angustifolia L., Poaceae, G
421. Poa bulbosa L. , Poaceae, H

422. Poa compressa L., Poaceae, $\mathrm{H}$

423. Poa pratensis L., Poaceae, G

424. Poa trivialis L., Poaceae, Ch

425. Poa trivialis L. ssp. sylvicola (Guss.) H.Lindb., Poaceae, H, LC

426. Polygala monspeliaca L., Polygalaceae, T

427. Polygala nicaeensis Risso ex Koch, Polygalaceae, $\mathrm{H}$

428. Polygala nicaeensis Risso ex Koch ssp. mediterranea Chodat, Polygalaceae, $\mathrm{H}$

429. Polygonum aviculare L., Polygonaceae, $T$

430. Polypodium vulgare L., Polypodiaceae, $\mathrm{H}$

431. Portenschlagiella ramosissima (Port.) Tutin, Apiaceae, H, E

432. Portulaca oleracea L., Portulacaceae, T

433. Potentilla argentea L., Rosaceae, $\mathrm{H}$

434. Potentilla australis Krašan, Rosaceae, $\mathrm{H}$

435. Potentilla heptaphylla L., Rosaceae, H

436. Potentilla hirta L., Rosaceae, H

437. Potentilla inclinata Vill., Rosaceae, $\mathrm{H}$

438. Potentilla micrantha Ramond ex DC., Rosaceae, H

439. Potentilla recta L., Rosaceae, $\mathrm{H}$

440. Primula veris L. ssp. columnae (Ten.) Ludi, Primulaceae, H, NT

441. Prunella laciniata L., Lamiaceae, H

442. Prunella vulgaris L., Lamiaceae, $\mathrm{H}$

443. Prunus avium L., Rosaceae, Ph

444. Prunus cerasus L., Rosaceae, $\mathrm{Ph}$

445. Prunus dulcis (Mill.) D. A. Webb, Rosaceae, Ph

446. Prunus mahaleb L., Rosaceae, Ph

447. Prunus spinosa L., Rosaceae, Ph

448. Pseudolysimachion barrelieri (Schott ex Roem. et Schult.) Holub, Scrophulariaceae, $\mathrm{H}$

449. Pteridium aquilinum (L.) Kuhn, Hypolepidaceae, G

450. Pulmonaria officinalis L., Boraginaceae, $\mathrm{H}$

451. Pulsatilla grandis Wender., Ranunculaceae, H, LC

452. Punica granatum L., Punicaceae, $\mathrm{N}$

453. Pyrus amygdaliformis Vill., Rosaceae, Ph

454. Quercus cerris L., Fagaceae, Ph

455. Quercus ilex L., Fagaceae, Ph

456. Quercus pubescens Willd., Fagaceae, Ph

457. Ranunculus acris L., Ranunculaceae, $\mathrm{H}$

458. Ranunculus arvensis L., Ranunculaceae, $T$

459. Ranunculus ficaria L., Ranunculaceae, G

460. Ranunculus illyricus L., Ranunculaceae, G

461. Ranunculus millefoliatus Vahl., Ranunculaceae, $\mathrm{H}$

462. Ranunculus muricatus $L$., Ranunculaceae, $T$

463. Reseda phyteuma L., Resedaceae, T

464. Rhagadiolus stellatus (L.) Gaertn., Cichoriaceae, T

465. Rhamnus intermedius Steud. et Hohst., Rhamnaceae, N, NT, E

466. Robinia pseudacacia L., Fabaceae, Ph, Inv

467. Rorippa lippizensis (Wulfen) Rchb., Brassicaceae, H, E

468. Rorippa sylvestris (L.) Besser, Brassicaceae, H

469. Rosa arvensis Huds., Rosaceae, N

470. Rosa canina L., Rosaceae, Ch

471. Rubia peregrina L., Rubiaceae, Ph

472. Rubus ulmifolius Schott., Rosaceae, N

473. Rumex acetosella L., Polygonaceae, $\mathrm{G}$

474. Rumex crispus L., Polygonaceae, $\mathrm{H}$

475. Ruscus aculeatus L., Liliaceae, Ch, LC

476. Ruta graveolens L., Rutaceae, Ch

477. Salvia officinalis L., Lamiaceae, Ch

478. Salvia pratensis L., Lamiaceae, H

479. Salvia sclarea L., Lamiaceae, T

480. Sambucus ebulus L., Caprifoliaceae, N

481. Sanguisorba minor Scop., Rosaceae, H

482. Satureja montana L., Lamiaceae, $\mathrm{Ch}$

483. Satureja subspicata Vis., Lamiaceae, Ch, LC

484. Saxifraga paniculata Mill., Saxifragaceae, H 
485. Saxifraga rotundifolia L., Saxifragaceae, H

486. Saxifraga tridactylites L., Saxifragaceae, T

487. Scandix pecten-veneris L., Apiaceae, $T$

488. Scilla autumnalis L., Liliaceae, $G$

489. Scilla bifolia L., Liliaceae, G

490. Scrophularia canina L., Scrophulariaceae, H

491. Sedum acre L., Crassulaceae, Ch

492. Sedum album L., Crassulaceae, Ch

493. Sedum ochroleucum Chaix, Crassulaceae, Ch

494. Sedum stellatum L., Crassulaceae, T

495. Sempervivum marmoreum Griseb., Crassulaceae, $\mathrm{Ch}$

496. Senecio jacobaea L., Asteraceae, H

497. Senecio vulgaris L., Asteraceae, H

498. Serratula radiata (Waldst. et Kit.) M.Bieb., Asteraceae, $H$

499. Seseli montanum L., Apiaceae, $H$

500. Seseli montanum L. ssp. tommasinii (Rchb. f.) Arcang., Apiaceae, H, E

501. Seseli pallasii Besser, Apiaceae, $\mathrm{H}$

502. Seseli tomentosum Vis., Apiaceae, H, NT, sE

503. Ses/eria autumnalis (Scop.) F. W. Schutz, Poaceae, H

504. Sesleria robusta Schott, Nyman et Kotschy, Poaceae, H

505. Sesleria tenuifolia Schrad., Poaceae, H

506. Sesleria tenuifolia Schrad. ssp. tenuifolia, Poaceae, H

507. Setaria viridis (L.) Beauv., Poaceae, $T$

508. Sideritis romana L., Lamiaceae, $\mathrm{T}$

509. Silene italica (L.) Pers., Caryophyllaceae, $\mathrm{H}$

510. Silene latifolia Poir. ssp. alba (Mill.) Greuter et Bourdet, Caryophyllaceae, H

511. Silene nutans L., Caryophyllaceae, $H$

512. Silene pendula L., Caryophyllaceae, T

513. Silene vulgaris (Moench) Garcke, Caryophyllaceae, H

514. Sisymbrium officinale (L.) Scop., Brassicaceae, T

515. Smyrnium perfoliatum L., Apiaceae, H

516. Solanum nigrum L., Solanaceae, T

517. Solanum villosum Mill., Solanaceae, T

518. Solidago gigantea Aiton, Asteraceae, G, Inv

519. Sonchus asper (L.) Hill, Cichoriaceae, T

520. Sorbus aria (L.) Crantz, Rosaceae, $\mathrm{Ph}$

521. Sorbus domestica L., Rosaceae, $\mathrm{Ph}$

522. Sorbus torminalis (L.) Crantz, Rosaceae, Ph

523. Sorghum halepense (L.) Pers, Poaceae, G

524. Spartium junceum L., Fabaceae, N

525. Spergula arvensis L., Caryophyllaceae, $T$

526. Spiraea cana Waldst. et Kit., Rosaceae, Ch, DD

527. Stachys germanica L., Lamiaceae, $\mathrm{H}$

528. Stachys maritima Gouan, Lamiaceae, $\mathrm{H}$

529. Stellaria media (L.) Vill., Caryophyllaceae, T

530. Stipa bromoides (L.) Dörfl., Poaceae, H

531. Stipa pennata L. ssp. eriocaulis (Borbás) Martinovský et Skalický, Poaceae, H

532. Tagetes minuta L., Asteraceae, T, Inv

533. Tamus communis L., Dioscoreaceae, $\mathrm{G}$

534. Tanacetum cinerariifolium (Trevir.) Sch. Bip., Asteraceae, H, E

535. Tanacetum corymbosum (L.) Sch.Bip., Asteraceae, H

536. Taraxacum officinale Weber, Cichoriaceae, $\mathrm{H}$

537. Teucrium arduini L., Lamiaceae, H, DD, E

538. Teucrium chamaedrys L., Lamiaceae, Ch

539. Teucrium montanum L., Lamiaceae, Ch

540. Teucrium polium L., Lamiaceae, Ch

541. Teucrium polium L. ssp. capitatum (L.) Arcang., Lamiaceae, Ch

542. Thalictrum aquilegifolium L., Ranunculaceae, $\mathrm{H}$

543. Thalictrum minus L., Ranunculaceae, $\mathrm{H}$

544. Thesium arvense Horv., Santalaceae, $H$

545. Thesium linophyllon L., Santalaceae, G

546. Thlaspi praecox Wulfen, Brassicaceae, Ch

547. Thymus bracteosus Vis. ex Benth., Lamiaceae, Ch, E

548. Thymus longicaulis C. Presl, Lamiaceae, Ch

549. Thymus striatus Vahl., Lamiaceae, Ch
550. Thymus vulgaris L., Lamiaceae, Ch

551. Tordylium apulum L., Apiaceae, T

552. Tordylium maximum L., Apiaceae, T

553. Torilis nodosa (L.) Gaertn., Apiaceae, $T$

554. Tragopogon dubius Scop. , Cichoriaceae, $H$

555. Trifolium alpestre L., Fabaceae, H

556. Trifolium angustifolium L., Fabaceae, $T$

557. Trifolium arvense L., Fabaceae, T

558. Trifolium aureum Pollich, Fabaceae, $T$

559. Trifolium campestre Schreber, Fabaceae, $T$

560. Trifolium dalmaticum Vis., Fabaceae, T, E

561. Trifolium incarnatum L., Fabaceae, $T$

562. Trifolium montanum L., Fabaceae, $H$

563. Trifolium nigrescens Viv., Fabaceae, $T$

564. Trifolium pratense L., Fabaceae, $\mathrm{H}$

565. Trifolium repens L., Fabaceae, Ch

566. Trifolium subterraneum $L .$, Fabaceae, $T$

567. Tulipa sylvestris L., Liliaceae, G, NT

568. Tussilago farfara L., Asteraceae, G

569. Ulmus minor Miller, Ulmaceae, Ph

570. Urtica dioica L., Urticaceae, H

571. Valantia muralis L., Rubiaceae, $\mathrm{T}$

572. Valeriana montana L., Valerianaceae, $H$

573. Valeriana officinalis L., Valerianaceae, $H$

574. Valeriana tuberosa L., Valerianaceae, $\mathrm{H}$

575. Valerianella locusta (L.) Laterrade, Valerianaceae, T

576. Valerianella rimosa Bastard, Valerianaceae, T

577. Verbascum chaixii Vill., Scrophulariaceae, H

578. Verbascum lanatum Schrad., Scrophulariaceae, H

579. Verbascum macrurum Ten., Scrophulariaceae, $\mathrm{H}$

580. Verbascum phoeniceum L., Scrophulariaceae, H

581. Veronica austriaca L. ssp. austriaca, Scrophulariaceae, H

582. Veronica chamaedrys L., Scrophulariaceae, H

583. Veronica persica Poir., Scrophulariaceae, T, Inv

584. Veronica teucrium L., Scrophulariaceae, Ch

585. Vicia cracca L., Fabaceae, H

586. Vicia faba L., Fabaceae, $T$

587. Vicia grandiflora Scop., Fabaceae, $T$

588. Vicia hybrida L., Fabaceae, T

589. Vicia lathyroides L., Fabaceae, $\mathrm{T}$

590. Vicia lutea L., Fabaceae, $T$

591. Vicia melanops Sm., Fabaceae, $\mathrm{T}$

592. Vicia narbonensis L., Fabaceae, T

593. Vicia ochroleuca Ten. ssp. dinara (K. Malý) Rohlena, Fabaceae, H, E

594. Vicia sativa L. , Fabaceae, T

595. Vicia tetrasperma (L.) Schreber, Fabaceae, $T$

596. Vincetoxicum hirundinaria Medik. , Asclepiadaceae, $H$

597. Vincetoxicum hirundinaria Medik. ssp. adriaticum (Beck) Markgr., Asclepiadaceae, $\mathrm{H}, \mathrm{E}$

598. Viola arvensis Murray, Violaceae, T

599. Viola odorata L., Violaceae, H

600. Viola tricolor L., Violaceae, T

601. Vitex agnus-castus L., Verbenaceae, $\mathrm{Ph}$

602. Vitis vinifera L. ssp. sylvestris (C. C. Gmel.) Hegi, Vitaceae, Ph, LC

603. Vulpia ciliata Dumort., Poaceae, $\mathrm{T}$

604. Xanthium strumarium L. ssp. italicum (Moretti) D. Löve, Poaceae, T, Inv

Abbreviations used for life forms: Ch (Chamaephyta), G (Geophyta), H (Hemicryptophyta), Ph (Phanerophyta), T (Therophyta); for IUCN categories: CR (Critically endangered), EN (Endangered), VU (Vulnerable), LC (Least Concern), NT (Near Threatened), DD (Data Deficient); for endemic status: endemic $(E)$, stenoendemic ( $\mathrm{SE}$ ); and for invasiveness (Inv).

Kratice koje se koriste za životne oblike: Ch (Chamaephyta), G (Geophyta), H (Hemicryptophyta), Ph (Phanerophyta), T (Therophyta); za IUCN kategorije: CR (kritično ugroženi), EN (ugroženi), VU (ranjivi), LC (najmanje zabrinjavajući), NT (gotovo ugroženi), DD (nedostatak podataka); za endemični status: endemski (E), stenoendemski (sE); i za invazivnost (Inv). 


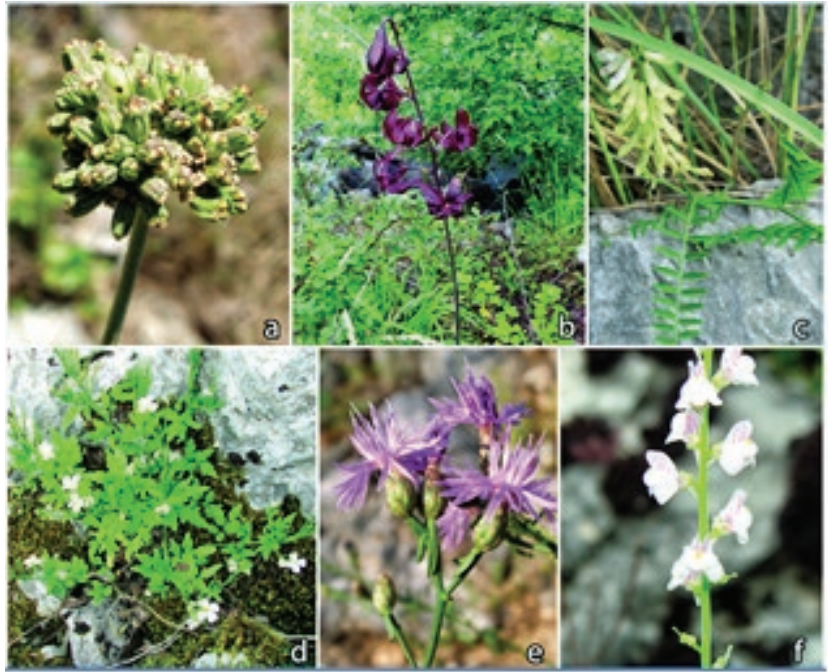

Figure 3. Some of endemic taxa of Matokit Mt: a) Seseli tomentosum Vis., b) Lilium martagon L. ssp. cattaniae (Vis.) Degen, c) Vicia ochroleuca Ten. ssp. dinara (K. Malý) Rohlena, d) Cardamine fialae Fritsch, e) Centaurea glaberrima Tau sch, f) Linaria microsepala A. Kern. (Photos by authors)

Slika 3. Neke endemične svojte planine Matokit: a) Seseli tomentosum Vis., b) Lilium martagon L. ssp. cattaniae (Vis.) Degen, c) Vicia ochroleuca Ten. ssp. dinara (K. Malý) Rohlena, d) Cardamine fialae Fritsch, e) Centaurea glaberrima Tausch, f) Linaria microsepala A. Kern. (autorske fotografije)

According to the Croatian Red List (Nikolić 2019b) a total of 36 taxa were found (Tab. 3). Two species (Papaver argemone and Consolida ajacis) are Critically Endangered (CR), one species (Hibiscus trionum) is Endangered (EN), while five species are classified as Vulnerable (VU). From the other IUCN categories, 16 taxa have been classified as Nearly Threatened (NT), 6 are of Least Concern (LC) and 6 were found to be Data Deficient (DD). Seventeen invasive and 32 endemic vascular plant taxa were recorded, of which 26 endemics in a broader sense and 6 stenoendemics (Tab. 3, Fig. 3).

The vascular flora of Matokit Mt was studied for the first time systematically and here is presented the first comprehensive floristic list of 604 vascular plant taxa, of different habitats, mostly grasslands in succession, rocky crevices and forest fringes, as well as a short overview on some interesting taxa in the flora Matokit Mt as a part of Biokovo massif. Floristic richness of the studied area is high, in comparison to several Croatian mountains along the eastern Adriatic coast especially considering the size of the area (Nikolić et al. 2008). The number of recorded plant taxa in comparison with similar floristic researches of the Dalmatian littoral mountains is quite similar. According to Kamenjarin (1996) in the flora of Kozjak Mt above Split 604 plant taxa were recorded, about 600 taxa were recorded in the flora of Mosor Mt (Bedalov and Šegulja 1987; Vladović and Ilijanić 1992, 1993, 1995). For Biokovo Mt the number of more than 1400 recorded taxa is result of more intensive and detailed floristic studies performed in the past (Hršak and Alegro 2008).
The occurrence of families Fabaceae (9.9\%), Poaceae (9.1\%), Asteraceae (7.4\%) and Lamiaceae (6.8\%) in the flora of Matokit Mt is quite normally distributed according to Nikolić (2001). The domination of hemicryptophytes (39.9\%) and therophytes (26.2\%) in the flora of Matokit Mt, as well as moderate present of geophytes (12.4\%), phanerophytes and chamaephytes $(10.8 \%)$ indicates high influence of the Mediterranean climate according to Horvat (1949).

In the flora of Matokit Mt a total of 32 endemic taxa were found (Tab. 3). Majority of endemic taxa were found within the vegetation of Natura 2000 grasslands and pastures in different succession stages e.g. a combination of rocky pastures of Chrysopogono grylli-Saturejion subspicatae Horvat \& Horvatić ex Černjavski, Grebenščikov \& Pavlović 1949 and grasslands of Saturejion subspicatae Tomić-Stanković 1970 , as well as within chasmophytic vegetation of the Centaureo cuspidatae-Portenschlagiellion ramosissimae Trinajstić ex Terzi et Di Pietro 2016. Especially interesting for the Croatian flora are the findings of recently recorded Cardamine fialae in the flora of Matokit Mt (Vukojević et al. 2016). The species grows on lower altitudes in rocky ground within the vegetation of forest fringes and secondary also in ruderal places, in arable fields, on shady screes and very rarely in rock crevices (Vukojević et al. 2016). All new localities in Croatia are close to Klobuk (locus classicus) in Bosnia and Herzegovina. Another Balkan endemic taxon, Erysimum croaticum, recently described by Polatschek (2013) from Zadvarje in Croatia was found in the flora of Matokit Mt there were a few individuals on screes of southern slopes. According to Polatschek (2013) this species is distributed in West Kosovo and Dalmatia (Mosor, Omiš, Brela region, Zadvarje and Makarska) and the new locality on Matokit Mt within Biokovo massif contributes to a better understanding of species distribution.

A spontaneous hybridogenic Iris population, evidently hybrid between I. illyrica and I. pseudopallida was recorded. Several populations with a few individuals growing within rocky grassland at higher altitudes (approximately 900$1000 \mathrm{~m}$ a.s.l.) were found. In the Flora Croatica Database (Nikolic 2019a) this taxon is not listed, and the occurrence of this Iris hybrid is not surprising because Matokit Mt represents the overlapping distribution area of I. illyrica and I. pseudopallida, and it could be found anywhere along the Adriatic littoral where parental species occur. Although those hybrids are known from literature in Croatian flora (Mitić et al. 2000, 2001, 2013; Biljaković 2002) taxonomical status of this hybrid is questionable and was not a subject of this research.

Some narrowly distributed species of the Croatian flora were also found in the flora of Matokit Mt e.g. Thymus bracteosus and Linaria microsepala. For endemic taxon Vicia ochroleuca ssp. dinara, the Matokit Mt represents the 
southernmost limit of its distribution. In addition, the northern slopes of Matokit Mt are the southernmost sites for the following taxa: Myrrhoides nodosa, Centaurea pannonica, and Saxifraga rotundifolia.

Within this floristic research we recorded 17 invasive plant taxa (Tab. 3) that were previously recorded by Vukojević (2011), Vukojević and Vitasović Kosić (2012). The tendency of uncontrolled spread indicates Ailanthus altissima and Ambrosia artemisiifolia, and limited expansion show: Amaranthus retroflexus, Xanthium strumarium ssp. italicum, Robinia pseudoacacia and Broussonetia papyrifera. All inventoried invasive species are in the habitat of abandoned arable land except for Robinia pseudoacacia, which spreads from the forest edge to the rocky grasslands. Some of the inventoried invasive plant species, are used as ornamental trees and shrubs in gardens in the studied area; e.g. Broussonenetia papyrifera, Robinia pseudoacacia, Phytolacca americana, Parthenocissus quinquefolia, Oenothera biennis, Solidago gigantea and Ailanthus altissima (Tab. 3). They could be replaced by planting attractive plant species, naturalized in the Mediterranean area, such as Celtis australis, Cercis siliquastrum and Ficus carica (Vukojević and Vitasović Kosić 2012).

The main problem in studied area is a high degree of vegetation degradation which is mostly present in lower zones (up to 400 meters). Because of strong human emigration from mountainous areas cattle-raising in northern settlements is almost completely abandoned (Vukosav 2006) and that endengered grassland habitats. Invasive species and grasslands in different stages of succession (mostly in a form of maquis and garrigue communities) occupy increasingly large areas mostly on abandoned arable land (Vukojević and Vitasović Kosić 2012). Grassland habitats succession and consequently the loss of endangered and endemic taxa contributes to the long-lasting succession of Juniperus oxycedrus L., which is a consequence of reduced grazing (sheep and goat) in this area.

During the year 2014 the reforestation of Aleppo pine ( $P i$ nus halepensis) was noticed. This management of reforestation resulted in an additional reduction of the grassland surface and significant loss of natural flora and vegetation, additionally threatening endemic and endangered taxa. Similarly, it has already happened in the nearby area of Orah Hill (on its southern slopes), that was afforested approximately 25 years ago, and already has today lost its natural grassland vegetation and flora diversity.

The flora diversity depends on human (non)activity (agriculture, urbanization). Therefore, proper management (regular mowing of meadows, pasture grazing, and maintenance of arable land) will help to create better conditions for the development of agriculture and sheep farming, while it can also reduce the spread of invasive plant taxa and contribute to conservation of plant diversity. In order to preserve the grassland habitats for endangered and endemic taxa we suggest the introduction of educational workshops for local residents and hunting associations.

To keep plant richness in this area, special attention in the future should be paid to its protection and conservation through management measures, such as maintaining a general low pressure of grazing by means of grazing rotation, to prevent the process of secondary succession and the spread of unpalatable competitive tall grasses at a landscape level (Vitasović Kosić et al. 2014). Here presented results are very important, because this is the first inventory of flora here, even though the area is a part of the Biokovo sensu lato and Dinaric mountains, which are known for extremely high plant diversity. Species inventories presented here are the basic information needed for conservation of flora and habitats. It also represents a background for future monitoring especially the decline of grasslands area, and harbouring habitats for rare species found on Matokit Mt. Therefore, we hope this information should become accessible for broader audience and future conservation planning.

\section{CONCLUSIONS ZAKLJUČCI}

The first detailed floristic study for Matokit Mt recorded a total of 604 vascular plant taxa on grasslands in different succession stages, rocky crevices and forest fringes habitats. Herbarium specimens (345 sheets) were collected, digitalized (206), deposited and are accessible in on-line ZAGR Herbarium. The results of this study point to the high plant richness in the study area as a result of various biotic and abiotic factors, conditioned further by geological variety, soil, topography and microclimatic factors. The Matokit Mt represents a new site for the Flora of Croatia, especially for endemic (32), and endangered (36) taxa. The occurrence of some very rare endemics (Erysimum croaticum and Cardamine fialae (until now the only known site in Croatia)) in the flora of Matokit Mt is of special interest for the national flora. In addition, the north slopes of the Matokit Mt are the southernmost findings for some taxa: Myrrhoides nodosa, Centaurea pannonica, and Saxifraga rotundifolia. The reduction of the area of rocky grasslands in the studied area as a result of the strong human emigration, rapid abandonment of agriculture and livestock breeding, reforestation of Pinus halepensis, and a long-lasting succession of Juniperus oxycedrus has been evident. Such loss of grassland habitats leads to endangerment of some protected (CR, EN, and VU) and endemic plant species, at the same time abandoning of agricultural habitats around Town Vrgorac. 


\section{ACKNOWLEDGEMENT}

\section{ZAHVALA}

Spetial thank to Mr. Aleksa Vukojević for the assistance on the field trip and for the hospitality during our stay in $\mathrm{Vr}$ gorac, Mrs Dragica Miletić for technical help in preparation of plant materials from Matokit Mt for ZAGR herbarium, sincere thank to Dr. Marija Jug-Dujaković for proofreading of English. We thank the reviewers for their careful reading of the manuscript and their constructive remarks.

\section{REFERENCES}

\section{LITERATURA}

- Bedalov M., Šegulja N., 1987: Prilog flori Mosora i njezina povezanost s Biokovom. Acta Biokovica. Radovi o prirodi biokov. područja 4: 175-188.

- Biljaković M., 2002: Varijabilnost plodova i sjemenki vrsta Iris illyrica Tomm. i Iris pseudopallida Trinastić (Iridaceae) na području Hrvatske. Diplomski rad, Sveučilište u Zagrebu, Prirodoslovno-matematički fakultet, Zagreb.

- Bogdanović S., Britvec M., Ljubičić I., Dujmović Purgar D., Vitasović Kosić I., 2016: Herbarium ZAGR of the Faculty of Agriculture (Zagreb, Croatia). Agric. Conspec. Sci. 81(1): 1-5.

- Boršić I., Milović M., Dujmović I., Bogdanović S., Cigić P, Rešetnik I., Nikolić T., Mitić B., 2008: Preliminary check-list of invasive alien plant species (IAS) in Croatia. Nat. Croat. 17(2):55-71.

- Domac R., 1957: Flora i vegetacija točila u primorskom pojasu Biokova. Biol. glas. Period. Biol. 10(1-2): 13-41.

- Horvat I., 1949: Nauka o biljnim zajednicama. Zagreb.

- Hršak V., Alegro A. L., 2008: Biljni svijet Biokova. In: Ozimec R. (ed.), Biokovo. Graphis d.o.o. i Javna ustanova "Park prirode Biokovo", 88-108.

- Kamenjarin J., 1996: Vascular Flora of Mount Kozjak above Split. Nat. Croat. 5(2): 119-144.

- Kušan F., 1956: Osobitosti u sastavu i rasporedu biljnog svijeta na planini Biokovu. Biološki glasnik 8, 103-109.

- Kušan F., 1969: Biljni pokrov Biokova. Zagreb

- Lovrić A. Ž., Rac M., 1987: Fitocenološka analiza vegetacije biokovskog područja. Acta Biokovica 4: 97-142.

- Martinović J., 2000: Tla u Hrvatskoj. Državna uprava za zaštitu prirode i okoliša, Zagreb.

- Mitić B., Nikolić T., Liber Z., 2000: Morphological and anatomical relationships in Alpine -Dinaric populations of the Genus Iris L., Pallidae series (A. Kern.) Trinajstić (Iridaceae). Acta Soc Bot Pol. 69(4): 285-291.

- Mitić B., Nikolić T., Liber Z., 2001: Morphological and karyological relationships within Alpine-Dinaric populations of the genus Iris L., Pallidae series (A. Kern.) Trinajstic (Iridaceae). Acta Soc. Bot. Pol. 70(3): 221-227

- Mitić B., Halbritter H., Šoštarić R., Nikolić T., 2013: Pollen morphology of the genus Iris L. (Iridaceae) from Croatia and surrounding area - taxonomic and phylogenetic implications. Plant Syst. Evol. 299(1): 271-288.
- Nikolić T., 2001: The diversity of Croatian vascular flora based on the Checklist and CROFlora database. Acta Bot. Croat. 60(1): 49-67.

- Nikolić T., 2006: Flora - Priručnik za inventarizaciju i praćenje stanja. Državni zavod za zaštitu prirode, Zagreb.

- Nikolić T., 2019a: Flora Croatica 4 - Vaskularna flora Republike Hrvatske. Alfa d.d., Zagreb.

- Nikolić T., ed., 2019b: Flora Croatica Database. University of Zagreb, Faculty of Science, Department of Botany (accessed: January 21,2019 )

- Nikolić T., Antonić O., Alegro A.L., Dobrović. I., Bogdanović S., Liber Z., Rešetnik I., 2008: Plant species diversity of Adriatic islands: An introductory survey. Pl. Biosyst.142(3): 435-44.

- Nikolić T., Mitić B., Boršić I., 2014: Flora hrvatske: invazivne biljke (Flora of Croatia: Invasive Plants). Alfa d.d., Zagreb.

- Nikolić T., Milović M., Bogdanović S., Jasprica N. 2015: Endemi u Hrvatskoj flori. Alfa d.d., Zagreb, pp 492

- Pavletić Z., 2002: Pregled florističkih istraživanja biokovskog područja. U: Kerovec M., Durbešić P., ur.: Prirodoslovna istraživanja biokovskog područja, Zbornik radova s kongresa 11.-16-10.1993. u Makarskoj. Knjiga 2. Ekološke monografije 5. Hrvatsko ekološko društvo, Zagreb, 1-12.

- Pignatti S., 1982, 1984: Flora d'Italia I-III. Edagricole, Bologna.

- Polatschek A., 2013: Revision der Gattung Erysimum (Cruciferae): Teil 5. Nord-, West-, Zentraleuropa, Rumänien und westliche Balkan-Halbinsel bis Albanien. Ann. Naturhist. Mus. Wien, B, 115:75-218.

- Rac M., Lovrić A. Ž., 1987: Prilog flori biokovskog područja, alge i vaskularna flora. Acta Biokovica IV: 31-46, Makarska.

- Radić J., 1974: Prilog poznavanju flore Biokova. Acta Bot. Croat. 33:219-229.

- Radić J., 1976: Bilje Biokova. Inst. „Planina i more”, Makarska.

- Radić J., 1977: Novi prilog poznavanju flore Biokova. Acta Bot. Croat. 36:173-175.

- Raunkiaer, 1934: The Life Forms of Plants and Statistical Plant Geography. Oxford University Press, London.

- Šilić Č., Šolić E. M., 1999: Contribution to the knowledge of the neophytic flora in the Biokovo area (Dalmatia, Croatia). Nat. Croat. 8(2): 109-116.

- Šilić, Č., Šolić, M. E., 2002: Addition to the vascular flora in the region of Biokovo (Dalmatia, Croatia), Nat. Croat. 11(3):341 363.

- Trinajstić I., 1987: Sintaksonomski pregled biljnih zajednica planine Biokovo. Acta Biokovica 4:143-174.

- Trinajstić I., 2002: Pregled vegetacije biokovskog područja. Ekološke monografije 5(2):13-37.

- Trinajstić I., 2008: Biljne zajednice Republike Hrvatske. Akademija šumarskih znanosti, Zagreb.

- Tutin T. G., Heywood V. H., Burges N. A., Valentine D. H., Walters S. M., Webb D. A., ur., 1964-1980, 1993: Flora Europaea 1-5. Cambridge University Press, United Kingdom.

- Vitasović Kosić I., Tardella F. M., Grbeša D., Škvorc Ž., Catorci A., 2014: Effects of abandonment on the functional composition and forage nutritive value of a North Adriatic dry grassland community (Ćićarija, Croatia). Appl. Ecol. Env. Res. 12(1):285-299.

- Vladović D., llijanić Lj., 1992: Prilog flori planine Mosor (Hrvatska). Acta Bot. Croat. 51:143-150.

- Vladović D., llijanić Lj., 1993: Drugi prilog flori planine Mosor (Hrvatska). Acta Bot. Croat. 52:145-152. 
- Vladović D., Ilijanić Lj., 1995: Treći prilog flori planine Mosor (Hrvatska). Acta Bot. Croat. 54: 41-46.

- Vukojević M., 2011: Vaskularna flora sjeverne padine planine Matokit i okolice Vrgorca. Diplomski rad, Sveučilište u Zagrebu, Agronomski fakultet, Zagreb.

- Vukojević M., Vitasović Kosić I., 2012: Mountain Matokit and Vrgorac city: a new localities of threatened and invasive plant taxa in Croatia. J. Cent. Eur. Agric. 13:150-166.
- Vukojević M., Vitasović Kosić I., Alegro A., Lakušić D., Bogdanović S., 2016: Cardamine fialae Fritsch (Brassicaceae) a new species in Croatian flora. Acta Bot. Croat. 75(2):213216.

- Vukosav B., 2006: Prostorna diferencijacija vrgoračkog područja na temelju krških prirodno-geografskih i društveno-geografskih obilježja. Odjel za geografiju, Sveučilište u Zadru, Geoadria $11 / 2,241-281$

\section{SAŽETAK}

Istraživana je vaskularna flora planine Matokita (Biokovo masiv) u južnoj Hrvatskoj u različitim vegetacijskim razdobljima od 2010.-2015., a pronađene su ukupno 604 vaskularne biljne svojte, iz 86 porodica i 337 rodova. Proučavano područje nikada nije istraženo u prošlosti i ovo su prvi detaljni floristički podaci za travnjake u različitim stadijima zarastanja. Sakupljeni herbarijski uzorci (345 listova) digitalizirani su i dostupni su na ZAGR herbariju on-line (http://herbarium.agr.hr). Dominantne porodice su mahunarke (Fabaceae 9,9\%), trave (Poaceae 9,1\%), glavočike cjevnjače (Asteraceae $7,4 \%$ ) i usnjače (Lamiaceae 6,8\%). Analiza životnih oblika pokazuje da su na planini Matokit dominantni hemikriptofiti $(39,9 \%)$ i terofiti $(26,2 \%)$ što ukazuje na veliki utjecaj mediteranske klime. Na istraživanom području zabilježeno je 36 ugroženih i 17 invazivnih biljnih svojti. Ukupno 32 biljne svojte su endemične (26 endema u širem smislu i 6 stenoendema) i predstavljaju nova nalazišta u flori Hrvatske. Pojava nekih vrlo rijetkih endema (Cardamine fialae Fritsch i Erysimum croaticum Polatschek) u flori Matokita od posebnog su interesa za nacionalnu floru.

KLJUČNE RIJEČI: biljna raznolikost, endemične vrste, sukcesija travnjaka, Vrgorac 